Research Article

\title{
Vibration Characteristics of Subway Tunnel Structure in Viscous Soil Medium
}

\author{
Dawei Ren, ${ }^{1,2}$ Shengjun Shao, ${ }^{1}$ Xiaoshan Cao ${ }^{D},{ }^{1}$ and Yifeng $\mathrm{Hu}^{1}$ \\ ${ }^{1}$ School of Civil Engineering and Architecture, Xi'an University of Technology, Xi'an 710048, China \\ ${ }^{2}$ School of Sciences, Xi'an University of Technology, Xi'an 710048, China \\ Correspondence should be addressed to Xiaoshan Cao; caoxsh@xaut.edu.cn
}

Received 28 December 2020; Revised 18 January 2021; Accepted 27 January 2021; Published 8 February 2021

Academic Editor: Yanhu Mu

Copyright (C) 2021 Dawei Ren et al. This is an open access article distributed under the Creative Commons Attribution License, which permits unrestricted use, distribution, and reproduction in any medium, provided the original work is properly cited.

Based on research on subway tunnels in a viscous soil medium, this paper establishes the vibration equation of a tunnel structure by using the theory of moderately thick cylindrical shells and the method of wave propagation. The soil around the tunnel is represented in simplified form as an isotropic viscoelastic medium to obtain the equation of motion of the soil, and the vibration control equation of the tunnel under the influence of viscous soil is obtained by coupling. By numerical calculations, the variation trends in the natural vibration frequency of the tunnel and attenuation affected by soil viscosity under different modes are given. Furthermore, the influences of the tunnel radius, wall thickness, and length on the vibration characteristics of a tunnel structure in viscous soil are discussed. This study will provide a reference for the design of subway vehicles and the antivibration design of subway tunnel structures.

\section{Introduction}

The influence of environmental vibrations caused by subway train operations is a problem that has attracted much attention, and many researchers have conducted a series of studies on this issue. These studies have mainly involved features such as the track system, tunnel structure, and physical characteristics of the surrounding strata. With the widespread use of shield technology in tunneling, a cylindrical shell structure has become the first choice for urban subway tunneling. The study of the natural vibration characteristics of underground structures, especially the calculation of their natural vibration frequency, is an important part of their dynamic analysis, which provides a theoretical basis for the dynamic calculation of structural design loads. At present, research on such structures mainly focuses on the theory of thin shells [1-4]. However, the thickness diameter ratio of subway tunnel structures is generally greater than $1: 12$, which does not conform to the assumptions of thin-shell theory.
Various studies have been performed to model ground-borne vibrations from underground railways using numerical and analytical methods. Moore and Guan [5] used continuous reflection to study the dynamic response of a tunnel under incident seismic waves, and their results showed that the interaction between soil and the tunnel was significant. Clouteau et al. $[6,7]$ established a three-dimensional model to calculate the dynamic interaction between a tunnel and soil, simulated the tunnel and soil with a finite element and a boundary element, respectively, and explained how the boundary element effectively simulated the periodic medium. The effectiveness of this method was demonstrated using two tunnels as examples. Forrest and Hunt $[2,3]$ proposed a pipe-in-pipe model and determined the dynamic response of a tunnel system in three-dimensional elastic soil by considering the tunnel-rock interaction. Gupta et al. [8] compared the pipe-in-pipe model and the periodic finite elementboundary element coupling model and analyzed the dynamic response of a tunnel lining in full space using these 
two models. Hussein et al. [9] developed a model for analyzing vibrations from railway tunnels in a half-space by using the pipe-in-pipe model. Then, Kuo et al. [10] proposed a pair of parallel-tunnel models in homogeneous full space and calculated the difference between the vertical and horizontal results of the double-tunnel model and the single-tunnel model. Clot et al. [11] extended this model to predict dynamic responses of a double-deck circular tunnel in full space. Parry et al. [12] verified the behavior of the pipelines in a software model of pipeline vibrations to determine ground noise and vibration levels above a construction tunnel. Yang et al. [13] used a centrifuge model experiment and numerical simulations to study the influence of changes in soil parameters with depth on vibrations caused by a tunnel. Via a comparison between experimental results and numerically simulated results, the validity of considering soil as a homogeneous medium in numerical simulations was illustrated.

Both numerical and analytical methods were used in the above studies to simulate the soil mass with a single medium. Therefore, other researchers used model of saturated porous media to simulate soil to increase the accuracy of the models. Senjuntichai et al. [14] studied timeharmonic vertical vibrations of an axisymmetric rigid foundation embedded in a homogeneous poroelastic soil. Kumar et al. [15] analyzed the radial displacement field of the solid phase and fluid phase when the surface of a cylindrical tunnel in saturated porous media was subjected to time-varying loads. Hasheminejad et al. $[16,17]$ studied the dynamic stress concentration in the area around the porous wall of a cylindrical cavity in an infinite saturated elastic medium under different vibrational modes and considered the dynamic response of the cavity lining and the surrounding soil under a moving load when the lining was not fully in contact with the surrounding saturated soil. Gao et al. [18] improved a 2.5-dimensional finiteelement model, and $\mathrm{He}$ et al. $[19,20]$ proposed a 2.5 -dimensional coupled finite element-boundary element model to simulate the three-dimensional dynamic interaction between saturated soil and a tunnel. Di et al. [21] presented a 3-dimensional multilayer model of a cylindrical tunnel for investigating train-induced dynamic stress in saturated soils, which considers multiple moving loads and the grouting layer of a shield tunnel. Lu and Jeng $[22,23]$ studied the dynamic response of fixed infinite cylindrical holes in porous media under axisymmetric ring loading and discussed the analytical determination of the dynamic response of porous media in half-space under moving point loading. On the basis of a fractional derivative model and the theory of saturated porous media, Gao and Wen [24] studied the dynamic characteristics of a viscoelastic soil fractional derivative viscoelastic lining system in the frequency domain under axisymmetric loading and fluid pressure. Yuan et al. $[25,26]$ determined the pore pressure transfer function of a tunnel in saturated soil, compared the equivalent model of elastic soil with the model of saturated soil, and studied the effect of pore fluids in the soil on the ground vibrations due to a tunnel buried in a layered half-space. In addition, some researchers have paid attention to the effect of the void between the soil and the tunnel interface $[27,28]$.

The above literature contains in-depth and detailed analyses of the vibrations caused by subway trains from multiple perspectives. However, although they are an essential parameter in vibration propagation, the vibration characteristics of tunnels buried in viscous soil have rarely been reported. In fact, the thickness-diameter ratio of most subway tunnels excavated by shield tunneling machines is greater than $1: 12$, and hence it is inappropriate to use thin-shell theory to model a tunnel in most studies. In addition, as a result of the natural complexity of soil, the soil around a tunnel is essentially an unsaturated porous medium. When the coupling between a tunnel and soil is considered, the influence of the soil medium on the natural vibration characteristics of the tunnel structure mainly comprises the influence of the additional mass and additional stiffness. Therefore, in this paper, the theory of moderately thick cylindrical shells is used to build a tunnel model, the Voigt-Kelvin equation is used to describe the constitutive relation of the soil medium, and the dispersion characteristic equation for the vibration characteristics of a tunnel in viscous soil is deduced by coupling of the tunnel and soil.

\section{Vibration Equation of Tunnel Structure}

The cylindrical coordinate system and a geometric model of the tunnel structure studied in this paper are shown in Figure 1. The tunnel structure is represented by the inner cylinder, and the soil around the tunnel is represented by the outer cylinder. In the figure, $L, R$, and $h$ represent the length, radius, and wall thickness of the tunnel, respectively; $u, v$, and $w$ represent the axial, tangential, and radial displacements of the tunnel surface, respectively; $x, \theta$, and $r$ denote the axial, tangential, and radial coordinates of the shell; $q_{x}$, $q_{\theta}$, and $q_{r}$ represent the external forces on the middle surface of the tunnel structure in the axial, tangential, and radial directions, respectively. On the basis of the theory of moderately thick shells [29], the equations of motion of the displacement components in the tunnel can be established as follows: 


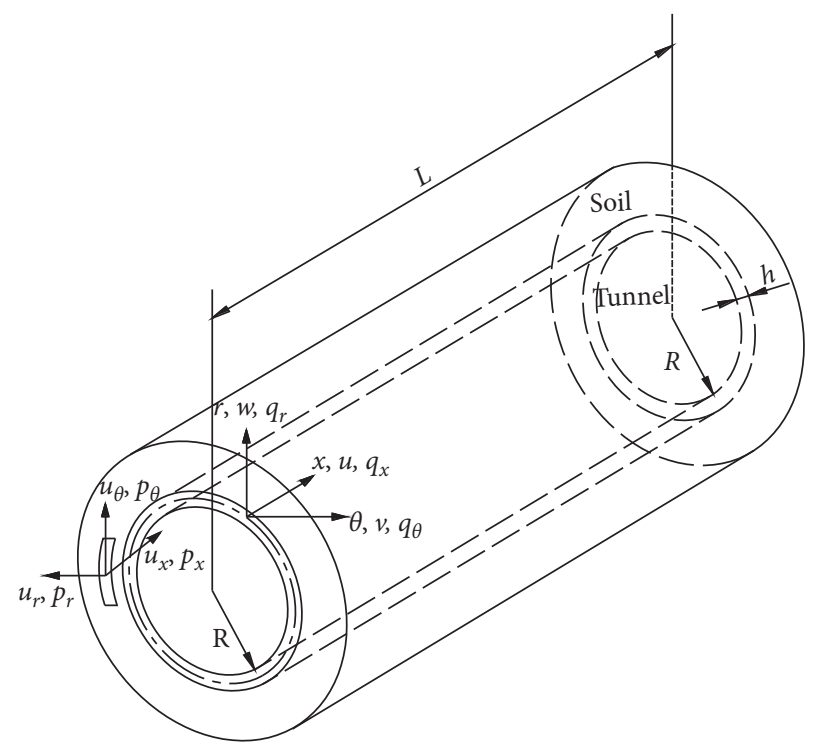

Figure 1: Geometric model of tunnel and soil.

$$
\begin{aligned}
& \frac{\partial^{2} u}{\partial x^{2}}+\left(\frac{1-v}{2 R^{2}}\right)\left(\frac{\partial^{2} u}{\partial \theta^{2}}\right)+\left(\frac{1+\nu}{2 R}\right)\left(\frac{\partial^{2} v}{\partial x \partial \theta}\right)+\left(\frac{\nu}{R}\right)\left(\frac{\partial w}{\partial x}\right)+\left(\frac{1}{K}\right) q_{x} \\
& +\left(\frac{h^{2} \varepsilon^{(a)}}{12 R}\right)\left(\frac{\partial^{2} \phi}{\partial x^{2}}-\left(\frac{1-\nu}{2 R^{2}}\right)\left(\frac{\partial^{2} \phi}{\partial \theta^{2}}\right)-\left(\frac{1-v}{2 R^{2}}\right)\left(\frac{\partial^{2} v}{\partial x \partial \theta}\right)\right)=\left(\frac{\rho J_{0}}{K}\right)\left(\frac{\partial^{2} u}{\partial t^{2}}\right), \\
& \left(\frac{1+v}{2 R}\right)\left(\frac{\partial^{2} u}{\partial x \partial \theta}\right)+\left(\frac{1-v}{2}\right)\left(\frac{\partial^{2} v}{\partial x^{2}}\right)+\left(\frac{1}{R^{2}}\right)\left(\frac{\partial^{2} v}{\partial \theta^{2}}\right)+\left(\frac{1}{R^{2}}\right)\left(\frac{\partial w}{\partial \theta}\right)+\left(\frac{q_{\theta}}{K}\right) \\
& +\left(\frac{h^{2} \varepsilon^{(a)}}{12 R}\right)\left(\left(\frac{1-v}{2}\right)\left(\frac{\partial^{2} \varphi}{\partial x^{2}}\right)-\left(\frac{1}{R^{2}}\right)\left(\frac{\partial^{2} \varphi}{\partial \theta^{2}}\right)\right)+\left(\frac{G h}{K R k_{\tau}}\right)\left(\varphi-\left(\frac{v}{R}\right)+\left(\frac{1}{R}\right)\left(\frac{\partial w}{\partial \theta}\right)\right)=\left(\frac{\rho J_{0}}{K}\right)\left(\frac{\partial^{2} v}{\partial t^{2}}\right), \\
& -\left(\frac{v}{R}\right)\left(\frac{\partial u}{\partial x}\right)-\left(\frac{1}{R^{2}}\right)\left(\frac{\partial v}{\partial \theta}\right)-\left(\frac{1}{R^{2}}\right) w+\left(\frac{G h}{K R k_{\tau}}\right)\left(\frac{\partial}{\partial \theta}\right)\left(\varphi-\frac{v}{R}+\left(\frac{1}{R}\right)\left(\frac{\partial w}{\partial \theta}\right)\right) \\
& +\left(\frac{h^{2} \varepsilon^{(a)}}{12 R^{3}}\right)\left(\frac{\partial \varphi}{\partial \theta}\right)+\left(\frac{G h}{K k_{\tau}}\right)\left(\frac{\partial}{\partial x}\right)\left(\phi+\frac{\partial w}{\partial x}\right)+\frac{q_{r}}{K}=\left(\frac{\rho J_{0}}{K}\right)\left(\frac{\partial^{2} w}{\partial t^{2}}\right), \\
& \frac{\partial^{2} \phi}{\partial x^{2}}+\left(\frac{1-\nu}{2 R^{2}}\right)\left(\frac{\partial^{2} \phi}{\partial \theta^{2}}\right)-\frac{m_{x}}{D}-\left(\frac{1}{D}\right)\left(\frac{G h}{k_{\tau}}\right)\left(\phi+\frac{\partial w}{\partial x}\right)+\left(\frac{1+\nu}{2 R}\right)\left(\frac{\partial^{2} \varphi}{\partial x \partial \theta}\right) \\
& +\frac{\varepsilon^{(a)}}{R}\left(\frac{\partial^{2} u}{\partial x^{2}}-\left(\frac{1-v}{2 R^{2}}\right)\left(\frac{\partial^{2} u}{\partial \theta^{2}}\right)\right)=\left(\frac{\rho J_{2}}{D}\right)\left(\frac{\partial^{2} \phi}{\partial t^{2}}\right) \text {, } \\
& \left(\frac{1-\nu}{2}\right)\left(\frac{\partial^{2} \varphi}{\partial x^{2}}\right)+\left(\frac{1}{R^{2}}\right)\left(\frac{\partial^{2} \varphi}{\partial \theta^{2}}\right)-\frac{G h}{D k_{\tau}}\left(\varphi-\frac{v}{R}+\left(\frac{1}{R}\right)\left(\frac{\partial w}{\partial \theta}\right)\right)-\frac{m_{\theta}}{D}+\left(\frac{1+\nu}{2 R}\right)\left(\frac{\partial^{2} \phi}{\partial x \partial \theta}\right) \\
& +\frac{\varepsilon^{(a)}}{R}\left(\left(\frac{1-v}{2}\right)\left(\frac{\partial^{2} v}{\partial x^{2}}\right)-\left(\frac{1}{R^{2}}\right)\left(\frac{\partial^{2} v}{\partial \theta^{2}}\right)-\left(\frac{1}{R^{2}}\right)\left(\frac{\partial w}{\partial \theta}\right)\right)=\left(\frac{\rho J_{2}}{D}\right)\left(\frac{\partial^{2} \varphi}{\partial t^{2}}\right) .
\end{aligned}
$$

In the above equations, Young's modulus of the shell material is denoted by $E$, the shear modulus is denoted by $G$, Poisson's ratio is denoted by $\nu$, and the density of the shell material is denoted by $\rho ; \phi$ and $\varphi$ represent the rotation of a point in the tunnel structure in the $x-r$ and $x-\theta$ planes, respectively; and $m_{x}$ and $m_{\theta}$ denote the external forces on the $x-r$ and $x-\theta$ plans, respectively; $k_{r}$ is the shear factor of the cylindrical shell; and $\varepsilon^{(a)}$ represents this item as an additional term, and $t$ is time, where $k_{r}=6 / 5, K=E h /\left(1-v^{2}\right)$, $D=E h^{3} / 12\left(1-v^{2}\right), J_{0}=h$, and $J_{2}=h^{3} / 12$. 
By using the wave propagation method, the solutions related to the displacement, the axial propagation wave number $k$, and the circumferential mode number $n$ can be obtained as follows:

$$
\begin{aligned}
u & =U \cos (n \theta) \exp [i(\omega t-k x)], \\
v & =V \sin (n \theta) \exp [i(\omega t-k x)], \\
w & =W \cos (n \theta) \exp [i(\omega t-k x)], \\
\phi & =\Phi \cos (n \theta) \exp [i(\omega t-k x)], \\
\varphi & =\Psi \sin (n \theta) \exp [i(\omega t-k x)],
\end{aligned}
$$

where $U, V, W, \Phi$, and $\Psi$ are the amplitudes of the displacement of the tunnel structure in the $x, \theta$, and $r$ directions and the $x-r$ and $x-\theta$ planes, respectively, and $\omega$ is the frequency. By substituting the form of equation (2) into equations (1a)-(1e), the vibration equation of the tunnel structure based on the theory of moderately thick cylindrical shells can be deduced by omitting the additional terms:

$$
[g][l] \bar{U}+[\bar{K}] \mathbf{q}=0 .
$$

In equation (3), $[\bar{K}]$ and $[g]$ are diagonal matrices of order $5,[l]$ is a coefficient matrix of order 5, $\bar{U}=(U, V, W, \Phi, \Psi)^{T}$, and $\mathbf{q}=\left(q_{x}, q_{\theta}, q_{r}, m_{x}, m_{\theta}\right)^{T}$, where the elements of each matrix are as follows:

$$
\begin{aligned}
& \bar{K}_{11}=\bar{K}_{22}=\bar{K}_{33}=\frac{1}{K}, \quad \bar{K}_{44}=\bar{K}_{55}=\frac{-1}{D}, \\
& g_{11}=\cos (n \theta) \exp [i(\omega t-k x)] \\
& g_{22}=\sin (n \theta) \exp [i(\omega t-k x)], \\
& g_{33}=\cos (n \theta) \exp [i(\omega t-k x)], \\
& g_{44}=\cos (n \theta) \exp [i(\omega t-k x)], \\
& g_{55}=\sin (n \theta) \exp [i(\omega t-k x)], \\
& l_{11}=-k^{2}-\frac{1-v}{2 R^{2}} n^{2}+\frac{\rho J_{0}}{K} \omega^{2}, \quad l_{12}=-\frac{1+v}{2 R} i k n=-l_{21}, \quad l_{13}=-i k \frac{v}{R}=-l_{31}, \quad l_{14}=l_{15}=0 \text {, } \\
& l_{22}=-\frac{1-v}{2} k^{2}-\frac{1}{R^{2}} n^{2}-\left(\frac{1}{K R^{2}}\right)\left(\frac{G h}{k_{\tau}}\right)+\frac{\rho J_{0}}{K} \omega^{2}, \quad l_{23}=-\frac{1}{R^{2}} n-\left(\frac{1}{K R^{2}}\right)\left(\frac{G h}{k_{\tau}}\right) n=l_{32}, \quad l_{24}=0, \quad l_{25}=\left(\frac{1}{K R}\right)\left(\frac{G h}{k_{\tau}}\right) \text {, } \\
& l_{33}=-\frac{1}{R^{2}}-\left(\frac{1}{K R^{2}}\right)\left(\frac{G h}{k_{\tau}}\right) n^{2}-\left(\frac{1}{K}\right)\left(\frac{G h}{k_{\tau}}\right) k^{2}+\frac{\rho J_{0}}{K} \omega^{2}, \quad l_{34}=-\left(\frac{1}{K}\right)\left(\frac{G h}{k_{\tau}}\right) i k, \quad l_{35}=\left(\frac{1}{K R}\right)\left(\frac{G h}{k_{\tau}}\right) n, \\
& l_{41}=l_{42}=0, \quad l_{43}=\left(\frac{1}{D}\right)\left(\frac{G h}{k_{\tau}}\right) i k, \quad l_{44}=-k^{2}-\frac{1-v}{2 R^{2}} n^{2}-\left(\frac{1}{D}\right)\left(\frac{G h}{k_{\tau}}\right)+\frac{\rho J_{2}}{D} \omega^{2}, \quad l_{45}=-\frac{1+v}{2 R} i k n=-l_{54}, \\
& l_{51}=0, \quad l_{52}=\left(\frac{1}{D R}\right)\left(\frac{G h}{k_{\tau}}\right), \quad l_{53}=\left(\frac{1}{D R}\right)\left(\frac{G h}{k_{\tau}}\right) n, \quad l_{55}=-\frac{1-v}{2} k^{2}-\frac{1}{R^{2}} n^{2}-\left(\frac{1}{D}\right)\left(\frac{G h}{k_{\tau}}\right)+\frac{\rho J_{2}}{D} \omega^{2} .
\end{aligned}
$$

\section{Equation of Motion of Soil}

If it is assumed that the soil around a tunnel is a homogeneous and isotropic linearly elastic medium, the equation of displacement for any point within the soil mass can be represented by the following Navier equation of motion [30]:

$$
(\lambda+\mu) \nabla \nabla \cdot \overline{\mathbf{u}}+\mu \nabla^{2} \overline{\mathbf{u}}+\rho_{s} \mathbf{f}=\rho_{s} \frac{\partial^{2} \overline{\mathbf{u}}}{\partial t^{2}} .
$$

In equation (5), $\bar{u}=\left(u_{r}, u_{\theta}, u_{x}\right)^{T}$ is the displacement vector of the soil mass, $\mathbf{f}$ is the vector of body forces, $\lambda=2 v_{s} G_{s} /\left(1-v_{s}\right)$, and $\mu=E_{s} / 2\left(1+v_{s}\right)$ are the Lamé constants of the medium (where $G_{s}$ is the shear modulus, $E_{s}$ is
Young's modulus, and $v_{s}$ is Poisson's ratio), $\rho_{s}$ is the density of the medium, and $\nabla$ is the Hamilton differential operator. In this case, the only body forces acting are those due to gravity; however, because the desired solution relates to vibration about an equilibrium position, these are ignored, and $\mathbf{f}$ is correspondingly set to zero.

If the soil around the tunnel structure is assumed to be a homogeneous and isotropic viscoelastic medium, the constitutive equation is represented by the Voigt-Kelvin equation for viscoelastic materials:

$$
s_{i j}=2 \mu e_{i j}+2 \eta \frac{\mathrm{d} e_{i j}}{\mathrm{~d} t},
$$


where $s_{i j}$ and $e_{i j}$ represent deviatoric stress and deviatoric strain, respectively, $\mu$ is the shear modulus, and $\eta$ is the coefficient of viscosity. If equation (6) is used to replace the constitutive equation of a linear elastomer $s_{i j}=2 \mu e_{i j}$, the equation of motion of the soil mass can be expressed as

$$
(\widetilde{\lambda}+\widetilde{\mu}) \nabla \nabla \cdot \overline{\mathbf{u}}+\widetilde{\mu} \nabla^{2} \overline{\mathbf{u}}+\rho_{s} f=\rho_{s} \frac{\partial^{2} \overline{\mathbf{u}}}{\partial t^{2}} .
$$

In equation $(7), \quad \tilde{\lambda}=\lambda+\bar{\lambda}(\partial / \partial t), \quad \tilde{\mu}=\mu+\bar{\mu}(\partial / \partial t)$, $\bar{\lambda}=-(2 / 3) \eta, \bar{\mu}=\eta$, and $(\partial / \partial t)$ represents the first partial derivative of the function with respect to time $t$.

According to the Stokes-Helmholtz vector decomposition theorem, the wave equation (7) can be solved by making use of the scalar potential $\Pi$ and the vector potential $\Omega$, and hence the displacement vector field can be expressed as follows:

$$
\overline{\mathbf{u}}=\nabla \Pi+\nabla \times \mathbf{\Omega},
$$

where $\Pi$ and $\Omega$ satisfy the following wave equations, respectively, in which $c_{p}$ and $c_{s}$ are the longitudinal wave velocity and shear wave velocity, respectively, in the soil medium.

$$
\nabla^{2} \Pi=\left(\frac{1}{c_{p}^{2}}\right)\left(\frac{\partial^{2} \Pi}{\partial t^{2}}\right), \quad \nabla^{2} \boldsymbol{\Omega}=\left(\frac{1}{c_{s}^{2}}\right)\left(\frac{\partial^{2} \boldsymbol{\Omega}}{\partial t^{2}}\right) .
$$

The Laplace equation for equation (9) above in a cylindrical coordinate system can be expressed as

$$
\begin{aligned}
\nabla^{2} \Pi= & \left(\frac{1}{r}\right)\left(\frac{\partial \Pi}{\partial r}\right)+\left(\frac{\partial^{2} \Pi}{\partial r^{2}}\right)+\left(\frac{1}{r^{2}}\right)\left(\frac{\partial^{2} \Pi}{\partial \theta^{2}}\right)+\frac{\partial^{2} \Pi}{\partial x^{2}}, \\
\nabla^{2} \boldsymbol{\Omega}= & \left(\nabla^{2} \Omega_{r}-\frac{\Omega_{r}}{r^{2}}-\left(\frac{2}{r^{2}}\right)\left(\frac{\partial \Omega_{\theta}}{\partial \theta}\right)\right) \mathbf{e}_{r} \\
& +\left(\nabla^{2} \Omega_{\theta}-\frac{\Omega_{\theta}}{r^{2}}+\left(\frac{2}{r^{2}}\right)\left(\frac{\partial \Omega_{r}}{\partial \theta}\right)\right) \mathbf{e}_{\theta}+\nabla^{2} \Omega_{x} \mathbf{e}_{x}
\end{aligned}
$$

where $\mathbf{e}_{x}, \mathbf{e}_{\theta}$, and $\mathbf{e}_{r}$ are unit vectors in the principal directions of the cylindrical coordinate system, and $\Omega_{x}, \Omega_{\theta}$, and $\Omega_{r}$ are the components of $\Omega$. Using the decomposition of equation (8), the components of the displacement of the soil medium can be expressed as

$$
\begin{aligned}
& u_{r}=\frac{\partial \Pi}{\partial r}+\left(\frac{1}{r}\right)\left(\frac{\partial \Omega_{x}}{\partial \theta}\right)-\frac{\partial \Omega_{\theta}}{\partial x}, \\
& u_{\theta}=\left(\frac{1}{r}\right)\left(\frac{\partial \Pi}{\partial \theta}\right)+\frac{\partial \Omega_{r}}{\partial x}-\frac{\partial \Omega_{x}}{\partial r}, \\
& u_{x}=\frac{\partial \Pi}{\partial x}+\left(\frac{1}{r}\right)\left(\frac{\partial\left(r \Omega_{\theta}\right)}{\partial r}\right)-\left(\frac{1}{r}\right)\left(\frac{\partial \Omega_{r}}{\partial \theta}\right) .
\end{aligned}
$$

The wave propagation solutions of the wave equation that satisfy equation (9) can be expressed as

$$
\begin{aligned}
\Pi & =f(r) \cos (n \theta) \exp [i(\omega t-k x)], \\
\Omega_{r} & =-i g_{r}(r) \sin (n \theta) \exp [i(\omega t-k x)], \\
\Omega_{\theta} & =-i g_{\theta}(r) \cos (n \theta) \exp [i(\omega t-k x)], \\
\Omega_{x} & =g_{x}(r) \sin (n \theta) \exp [i(\omega t-k x)] .
\end{aligned}
$$

These represent harmonic solutions in the same way as those used in the analysis of the cylindrical shell, but now there are also variations with the radius $r$, which are governed by the functions $f, g_{r}, g_{\theta}$, and $g_{x}$, respectively. Here, $\omega$ is the frequency in the complex domain, of which the real part is the vibration frequency and the imaginary part is related to attenuation. Substitution of the solutions expressed by equation (12) into equation (9), making use of the definitions expressed by equation (10) and considering each component of the equation in $\Omega$ in turn, results in the four following differential equations:

$$
\begin{gathered}
\frac{\mathrm{d}^{2} f}{\mathrm{~d} r^{2}}+\left(\frac{1}{r}\right)\left(\frac{\mathrm{d} f}{\mathrm{~d} r}\right)-\left[\frac{n^{2}}{r^{2}}-\left(\frac{\omega^{2}}{c_{p}^{2}}-k^{2}\right)\right] f=0, \\
\frac{\mathrm{d}^{2} g_{r}}{\mathrm{~d} r^{2}}+\left(\frac{1}{r}\right)\left(\frac{\mathrm{d} g_{r}}{\mathrm{~d} r}\right)+\frac{1}{r^{2}}\left(-n^{2} g_{r}+2 n g_{\theta}-g_{r}\right)-k^{2} g_{r}+\left(\frac{\omega^{2}}{c_{s}^{2}}\right) g_{r}=0, \\
\frac{\mathrm{d}^{2} g_{\theta}}{\mathrm{d} r^{2}}+\left(\frac{1}{r}\right)\left(\frac{\mathrm{d} g_{\theta}}{\mathrm{d} r}\right)+\frac{1}{r^{2}}\left(-n^{2} g_{\theta}+2 n g_{r}-g_{\theta}\right)-k^{2} g_{\theta}+\left(\frac{\omega^{2}}{c_{s}^{2}}\right) g_{\theta}=0, \\
\frac{\mathrm{d}^{2} g_{x}}{\mathrm{~d} r^{2}}+\left(\frac{1}{r}\right)\left(\frac{\mathrm{d} g_{x}}{\mathrm{~d} r}\right)-\left[\frac{n^{2}}{r^{2}}-\left(\frac{\omega^{2}}{c_{s}^{2}}-k^{2}\right)\right] g_{x}=0 . \\
\frac{\mathrm{d}^{2} g_{r}}{\mathrm{~d} r^{2}}+\left(\frac{1}{r}\right)\left(\frac{\mathrm{d} g_{r}}{\mathrm{~d} r}\right)-\left[\frac{(n+1)^{2}}{r^{2}}-\left(\frac{\omega^{2}}{c_{s}^{2}}-k^{2}\right)\right] g_{r}=0 .
\end{gathered}
$$

According to the property of gauge invariance [31], defining $g_{r}=-g_{\theta}$ and substituting into the second and third 
The first and fourth forms of equation (13) are modified Bessel equations of order $n$, and equation (14) is a modified Bessel equation of order $(n+1)$. From the boundary conditions at an infinite distance, $f, g_{r}, g_{\theta}$, and $g_{x}$ are expressed as

$$
\begin{aligned}
f(r) & =A W_{n}\left(\xi_{1} r\right), \\
g_{r}(r) & =-g_{\theta}(r)=B W_{n+1}\left(\xi_{2} r\right), \\
g_{x}(r) & =C W_{n}\left(\xi_{2} r\right),
\end{aligned}
$$

where $A, B$, and $C$ are undetermined constants; $W_{n}$ and $W_{n+1}$ represent a third class of Bessel functions of orders $n$ and $n+1$, respectively; and $\xi_{1}=\sqrt{\omega^{2} /\left(c_{p}^{2}-k^{2}\right)}$ and $\xi_{2}=\sqrt{\omega^{2} /\left(c_{s}^{2}-k^{2}\right)}$ are the radial components of the longitudinal wave number and the shear wave number in the soil medium, respectively.

By substituting equations (12) and (15) into equation (11), expressions for axial, tangential, and radial displacements can be obtained:

$$
\begin{aligned}
& u_{r}=\left[\xi_{1} A W_{n}^{\prime}\left(\xi_{1} r\right)-k B W_{n+1}\left(\xi_{2} r\right)+\frac{n}{r} C W_{n}\left(\xi_{2} r\right)\right] \cos (n \theta) \exp [i(\omega t-k x)], \\
& u_{\theta}=\left[-\frac{n}{r} A W_{n}\left(\xi_{1} r\right)-k B W_{n+1}\left(\xi_{2} r\right)-\xi_{2} C W_{n}^{\prime}\left(\xi_{2} r\right)\right] \sin (n \theta) \exp [i(\omega t-k x)], \\
& u_{x}=\left[-i k A W_{n}\left(\xi_{1} r\right)+i \xi_{2} B W_{n+1}^{\prime}\left(\xi_{2} r\right)+i \frac{n+1}{r} B W_{n+1}\left(\xi_{2} r\right)\right] \cos (n \theta) \exp [i(\omega t-k x)] .
\end{aligned}
$$

From the relationship between stress and strain, expressions for stresses $\sigma$ in the soil medium can be obtained:

$$
\begin{aligned}
& \left(\begin{array}{c}
\sigma_{r r} \\
\sigma_{r \theta} \\
\sigma_{r x}
\end{array}\right)=\left(\begin{array}{ccc}
\cos (n \theta) & 0 & 0 \\
0 & \sin (n \theta) & 0 \\
0 & 0 & \cos (n \theta)
\end{array}\right)\left(\begin{array}{ccc}
d_{11} & d_{12} & d_{13} \\
d_{21} & d_{22} & d_{23} \\
d_{31} & d_{32} & d_{33}
\end{array}\right)\left(\begin{array}{c}
A \\
B \\
C
\end{array}\right) \cdot \exp [i(\omega t-k x)], \\
& d_{11}=(\tilde{\lambda}+2 \widetilde{\mu}) \xi_{1}^{2} W_{n}^{\prime \prime}\left(\xi_{1} r\right)+\tilde{\lambda} \frac{\xi_{1}}{r} W_{n}^{\prime}\left(\xi_{1} r\right)-\tilde{\lambda}\left(\frac{n^{2}}{r^{2}}+k^{2}\right) W_{n}\left(\xi_{1} r\right), \\
& d_{12}=-2 \widetilde{\mu} k \xi_{2} W_{n+1}^{\prime}\left(\xi_{2} r\right), \\
& d_{13}=2 \widetilde{\mu}\left[\frac{n_{n}}{r} \xi_{2} W_{n}^{\prime}\left(\xi_{2} r\right)-\frac{n}{r^{2}} W_{n}\left(\xi_{2} r\right)\right] \\
& d_{21}=2 \widetilde{\mu}\left[\frac{n}{r^{2}} W_{n}\left(\xi_{1} r\right)-\frac{n}{r} \xi_{1} W_{n}^{\prime}\left(\xi_{1} r\right)\right] \text {, } \\
& d_{22}=\tilde{\mu}\left[\frac{n+1}{r} k W_{n+1}\left(\xi_{2} r\right)-k \xi_{2} W_{n+1}^{\prime}\left(\xi_{2} r\right)\right], \\
& d_{23}=\widetilde{\mu}\left[\frac{1}{r} \xi_{2} W_{n}^{\prime}\left(\xi_{2} r\right)-\frac{n^{2}}{r^{2}} W_{n}\left(\xi_{2} r\right)-\xi_{2}^{2} W_{n}^{\prime \prime}\left(\xi_{2} r\right)\right] \text {, } \\
& d_{31}=-2 i k \tilde{\mu} \xi_{1} W_{n}^{\prime}\left(\xi_{1} r\right), \\
& d_{32}=\tilde{\mu}\left[\left(i k^{2}-i \frac{n+1}{r^{2}}\right) W_{n+1}\left(\xi_{2} r\right)+i \xi_{2} \frac{n+1}{r} W_{n+1}^{\prime}\left(\xi_{2} r\right)+i \xi_{2}^{2} W_{n+1}^{\prime \prime}\left(\xi_{2} r\right)\right] \text {, } \\
& d_{33}=-i k \tilde{\mu} \frac{n}{r} W_{n}\left(\xi_{2} r\right) \text {. }
\end{aligned}
$$




\section{Vibration Control Equation of Tunnel in Viscous Soil}

It is assumed that, on the contact surface, the surface displacement of the tunnel structure (cylindrical shell) is equal to that of the soil medium. When $r=R+(h / 2)$,

$$
\begin{array}{r}
U \cos (n \theta) \exp [i(\omega t-k x)]+\Phi \cos (n \theta) \exp [i(\omega t-k x)] \cdot \frac{h}{2}=\left.u_{x}\right|_{r=R+(h / 2)} \\
V \sin (n \theta) \exp [i(\omega t-k x)]+\Psi \sin (n \theta) \exp [i(\omega t-k x)] \cdot \frac{h}{2}=\left.u_{\theta}\right|_{r=R+(h / 2)} \\
W \cos (n \theta) \exp [i(\omega t-k x)]=\left.u_{r}\right|_{r=R+(h / 2)} .
\end{array}
$$

By substituting equation (16) into equation (18), the displacement conditions expressed by the matrix for viscoelastic medium can be obtained:

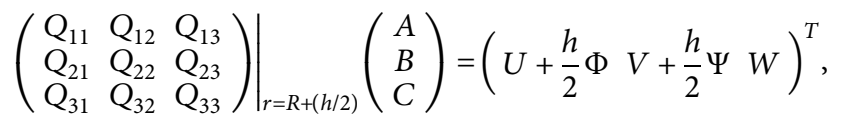

where $Q_{11}=-i k W_{n}\left(\xi_{1} r\right), Q_{12}=i \xi_{2} W_{n+1}^{\prime}\left(\xi_{2} r\right)+i(n+1 / r)$ $W_{n+1}\left(\xi_{2} r\right), \quad Q_{13}=0, \quad Q_{21}=-(n / r) W_{n}\left(\xi_{1} r\right), \quad Q_{22}=$ $-k W_{n+1}\left(\xi_{2} r\right), \quad Q_{23}=-\xi_{2} W_{n}^{\prime}\left(\xi_{2} r\right)$, and $Q_{31}=\xi_{1} W_{n}^{\prime}\left(\xi_{1} r\right)$, $Q_{32}=-k W_{n+1}\left(\xi_{2} r\right), \quad Q_{33}=(n / r) W_{n}\left(\xi_{2} r\right)$. According to the continuous condition of the force on the contact surface, the force $F$ exerted by the soil medium on the outer surface of the tunnel structure can be determined as follows:

$$
\left(\begin{array}{llll}
F_{x} & F_{\theta} & F_{r}
\end{array}\right)^{T}=\left(\begin{array}{lll}
\left.\sigma_{r x}\right|_{r=R+(h / 2)} & \left.\sigma_{r \theta}\right|_{r=R+(h / 2)} & \left.\sigma_{r r}\right|_{r=R+(h / 2)}
\end{array}\right)^{T} .
$$

By substituting equation (17) into equation (18), the following continuous conditions can be obtained:

$$
\mathbf{F}=\left(\begin{array}{c}
F_{x} \\
F_{\theta} \\
F_{r}
\end{array}\right)=\left[g^{\prime}\right]\left(\begin{array}{lll}
\alpha_{11} & \alpha_{12} & \alpha_{13} \\
\alpha_{21} & \alpha_{22} & \alpha_{23} \\
\alpha_{31} & \alpha_{32} & \alpha_{33}
\end{array}\right)_{r=R+(h / 2)}\left(\begin{array}{c}
A \\
B \\
C
\end{array}\right),
$$

where $\alpha_{11}=-2 i \tilde{\mu} k \xi_{1} W_{n}^{\prime}\left(\xi_{1} r\right), \quad \alpha_{12}=i \widetilde{\mu} \quad\left[\left(k^{2}-\left(n+1 / r^{2}\right)\right)\right.$ $\left.W_{n+1}\left(\xi_{2} r\right)+\xi_{2}(n+1 / r) W_{n+1}^{\prime}\left(\xi_{2} r\right)+\xi_{2}^{2} W_{n+1}^{\prime \prime}\left(\xi_{2} r\right)\right], \quad \alpha_{13}=$ $-i \tilde{\mu} k(n / r) W_{n}\left(\xi_{2} r\right), \quad \alpha_{21}=2 \widetilde{\mu}\left[\left(n / r^{2}\right) W_{n}\left(\xi_{1} r\right)-(n / r) \xi_{1} W_{n}^{\prime}\right.$ $\left.\left(\xi_{1} r\right)\right], \alpha_{22}=\tilde{\mu}\left[(n+1 / r) k W_{n+1}\left(\xi_{2} r\right)-k \xi_{2} W_{n+1}^{\prime}\left(\xi_{2} r\right)\right], \alpha_{23}=$ $\tilde{\mu}\left[\left(\xi_{2} / r\right) W_{n}^{\prime}\left(\xi_{2} r\right)-\left(n^{2} / r^{2}\right) W_{n}\left(\xi_{2} r\right)-\xi_{2}^{2} W_{n}^{\prime \prime}\left(\xi_{2} r\right)\right], \quad \alpha_{31}=$ $(\widetilde{\lambda}+2 \widetilde{\mu}) \xi_{1}^{2} W_{n}^{\prime \prime}\left(\xi_{1} r\right)+\widetilde{\lambda}\left(\xi_{1} / r\right) W_{n}^{\prime}\left(\xi_{1} r\right)-\widetilde{\lambda}\left(\left(n^{2} / r^{2}\right)+k^{2}\right) W_{n}$ $\left(\xi_{1} r\right), \quad \alpha_{32}=-2 \widetilde{\mu} k \xi_{2} W_{n+1}^{\prime}\left(\xi_{2} r\right), \quad \alpha_{33}=2 \widetilde{\mu}\left[(n / r) \xi_{2} \quad W_{n}^{\prime}\left(\xi_{2} r\right)\right.$ $\left.-\left(n / r^{2}\right) W_{n}\left(\xi_{2} r\right)\right]$, and $\left[g^{\prime}\right]=\operatorname{diag}\left\{\begin{array}{lll}g_{11} & g_{22} & g_{33}\end{array}\right\}$.

Substituting equation (19) into equation (21) gives

$$
\mathbf{F}=\left[g^{\prime}\right][\alpha][\beta] \overline{U^{\prime}},
$$

where $\quad \overline{\mathbf{U}}^{\prime}=(U+(h / 2) \Phi, \quad V+(h / 2) \Psi, \quad W)^{T}, \quad[\alpha]=$ $\left.\left(\begin{array}{lll}\alpha_{11} & \alpha_{12} & \alpha_{13} \\ \alpha_{21} & \alpha_{22} & \alpha_{23} \\ \alpha_{31} & \alpha_{32} & \alpha_{33}\end{array}\right)\right|_{r=R+(h / 2)} \quad$ and $\quad[\beta]=\left(\begin{array}{lll}\beta_{11} & \beta_{12} & \beta_{13} \\ \beta_{21} & \beta_{22} & \beta_{23} \\ \beta_{31} & \beta_{32} & \beta_{33}\end{array}\right)=$ $\left.\left(\begin{array}{lll}Q_{11} & Q_{12} & Q_{13} \\ Q_{21} & Q_{22} & Q_{23} \\ Q_{31} & Q_{32} & Q_{33}\end{array}\right)^{-1}\right|_{r=R+(h / 2)}$. By extending $\mathbf{F},[g],[\alpha],[\beta]$, and $\bar{U}^{\prime}$ in equation (22), we can obtain

$$
\bar{F}=[g][\bar{\alpha}][\bar{\beta}][\bar{h}] \bar{U} .
$$

In equation (23), $\bar{F}=\left(F_{x}, F_{\theta}, F_{r}, 0,0\right)^{T}, \bar{U}=(U, V$, $W, \Phi, \Psi)^{T}, \quad[\bar{\alpha}]_{5 \times 5}=\left(\begin{array}{cc}\alpha & 0_{3 \times 2} \\ 0_{2 \times 3} & 0_{2 \times 2}\end{array}\right), \quad[\bar{\beta}]_{5 \times 5}=\left(\begin{array}{cc}\beta & 0_{3 \times 2} \\ 0_{2 \times 3} & 0_{2 \times 2}\end{array}\right)$, $[\bar{h}]_{5 \times 5}=\left(\begin{array}{cc}I_{3} & h_{0} I_{2} \\ 0_{2 \times 3} & 0_{2 \times 2}\end{array}\right), I_{k}$ is the identity matrix of order $k$, and $h_{0}=(h / 2)$.

In accordance with equation (23) for the continuous condition of the force on the contact surface, the force exerted by the soil medium on the surface of the tunnel can be determined by the translation of force:

$$
\mathbf{q}=[\bar{h}]^{T} \bar{F}=[\bar{h}]^{T}[g][\bar{\alpha}][\bar{h}] \bar{U} .
$$

By substituting equation (24) into equation (3), the vibration control equation of a tunnel structure in viscous soil based on the theory of moderately thick shells can be obtained:

$$
\left([l]+[\bar{K}][\bar{h}]^{T}[\bar{\alpha}][\bar{\beta}][\bar{h}]\right) \bar{U}=0 .
$$

For the nonzero solution of equation (25), the determinant of its coefficient matrix must be equal to zero, and thus the dispersion characteristic equation for the vibration characteristics of the tunnel can be obtained:

$$
\left|[l]+[\bar{K}][\bar{h}]^{T}[\bar{\alpha}][\bar{\beta}][\bar{h}]\right|=0 .
$$




\section{Numerical Results}

5.1. Boundary Conditions. In this paper, the simplified boundary conditions in the form of solid supports at both ends of the tunnel are selected for calculation. The parameters of the tunnel are selected as follows: $E=50 \mathrm{GPa}$, $\nu=0.3, \rho=2500 \mathrm{~kg} / \mathrm{m}^{3}, h=0.25 \mathrm{~m}, R=2.75 \mathrm{~m}, \lambda=28.8 \mathrm{GPa}$, and $\mu=19.2 \mathrm{GPa}$. The parameters of the soil around the tunnel are chosen as follows: $E_{s}=400 \mathrm{MPa}, v_{s}=0.35$, and $\rho_{s}=1960 \mathrm{~kg} / \mathrm{m}^{3}$. The viscosity coefficient of the soil is denoted by $\eta$ and has units of Pa.s.

In accordance with the simplified treatment of boundary conditions in the case of cylindrical shells in an infinite flow field [1], the boundary conditions at both ends of the tunnel are approximately treated as solid beams in this paper, and it is considered that the wave number for waves propagated in the axial direction is related to the boundary conditions at both ends of the cylindrical shell. The tunnel is represented in simplified form as a cylindrical shell with fixed supports at both ends, and hence $k L=(2 m+1) \pi / 2$, where $m$ is the axial mode number. From the wave number $k$ and the circumferential mode number $n$, the characteristic equation (26) can be used to calculate the frequency of the cylindrical shell under different modes $(m, n)$ in the complex domain. Here, the circular frequency $\omega$ is a complex number, of which the real part represents the natural vibration frequency of the tunnel and the imaginary part is related to attenuation. Circular frequency $\omega$ and frequency $f$ satisfy $\omega=2 \pi f$, where $f$ is a complex number, $f=f_{R}+i f_{\mathrm{Im}}$, the real part $f_{R}$ represents the vibration frequency of the tunnel, and the imaginary part $f_{\mathrm{Im}}$ is related to attenuation. To assess the influence of soil viscosity on attenuation at the vibration frequency of the tunnel structure, the attenuation coefficient $\eta^{*}$ is introduced and is defined as

$$
\eta^{*}=\exp \left(2 \pi \cdot \frac{f_{R}}{f_{\mathrm{Im}}}\right)
$$

5.2. Effect of Soil Viscosity on Frequency of Tunnel Structure. Figure 2 shows the variations in the natural frequency of each mode at different soil viscosities. When $\eta=0$, the viscous soil around the tunnel becomes isotropic and linearly elastic. With an increase in $\eta$, the natural frequencies of the tunnel structure are reduced. The results show that the influence of a viscous soil medium on the vibration characteristics of the tunnel structure is mainly manifested as the influence of additional mass.

Figure 3 shows the variations in the attenuation coefficient $\eta^{*}$ of the tunnel for each mode at different soil viscosity. The attenuation coefficient increases significantly with an increase in soil viscosity. This indicates that viscous soil surrounding the tunnel has a strong influence on energy dissipation. In addition, with an increase in soil viscosity, the attenuation coefficient increases to a significantly greater extent for the high-order modes than for the low-order modes, which explains why vibrations at the natural frequency of the tunnel, as shown in Figure 2, are attenuated more significantly in the case of high-order modes at high viscosity.

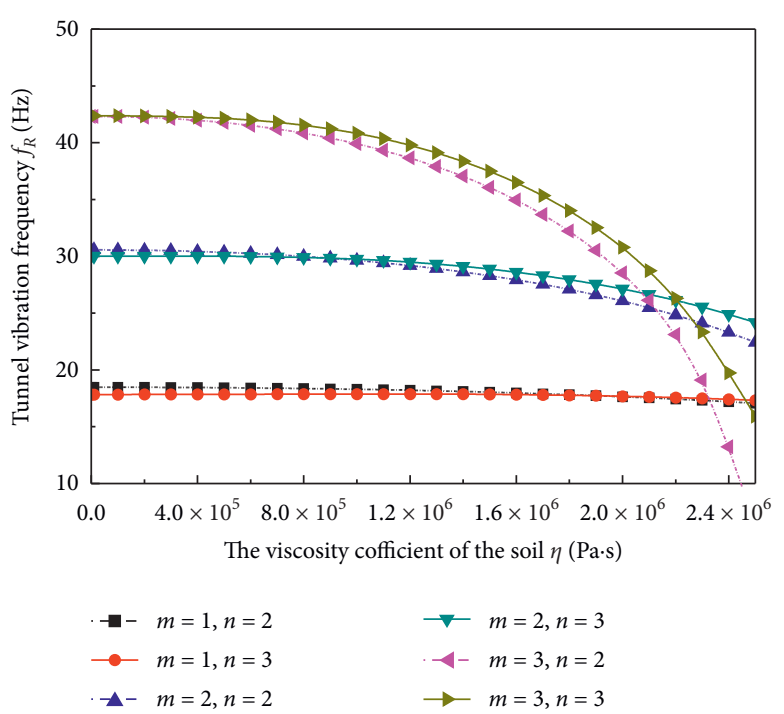

FIGURE 2: Relationship between natural frequency of the tunnel and changes in soil viscosity for different modes.

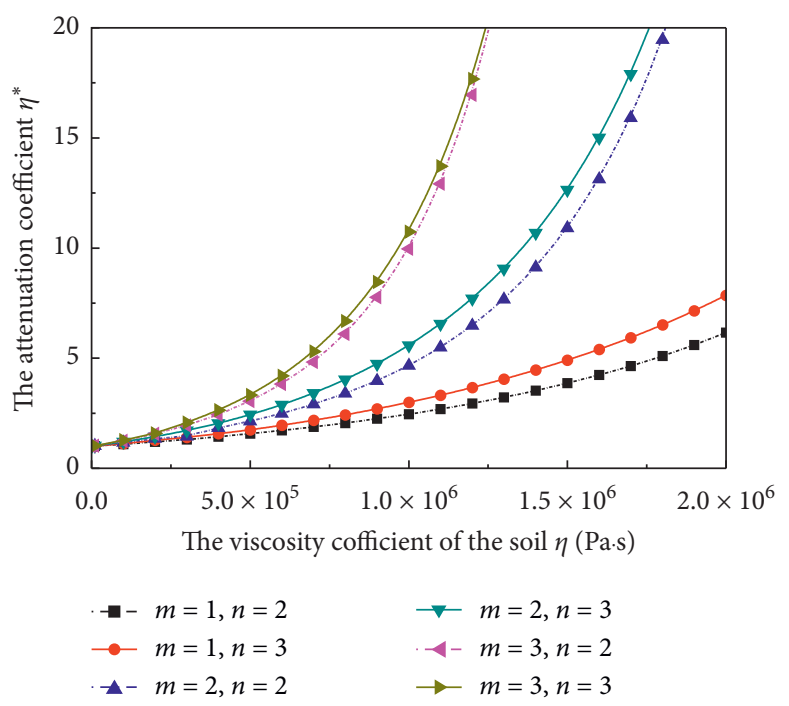

Figure 3: Relationship between attenuation coefficient and changes in soil viscosity for different modes.

\subsection{Effect of Tunnel Radius on Frequency of Tunnel Structure.} In order to analyze the influence of the tunnel radius on the natural vibration frequency of the tunnel structure under the influence of viscous soil, other calculation parameters remain unchanged, while the tunnel radius is varied, and the viscosity coefficient of the soil mass is defined as $\eta=1.0 \times 10^{6} \mathrm{~Pa} \cdot \mathrm{s}$.

As shown in Figure 4, the natural frequency of the tunnel is basically the same at the same axial mode number $m$ for each mode. With an increase in the tunnel radius, the natural frequency of the tunnel for each mode slight increases. Figure 5 shows the attenuation curves for each mode corresponding to Figure 4. At the same axial mode number, the attenuation coefficient for a high circumferential mode number is higher when the tunnel radius is less than $0.3 \mathrm{~m}$. On the whole, it can be seen that the radius has little influence on the natural frequency of the tunnel. 


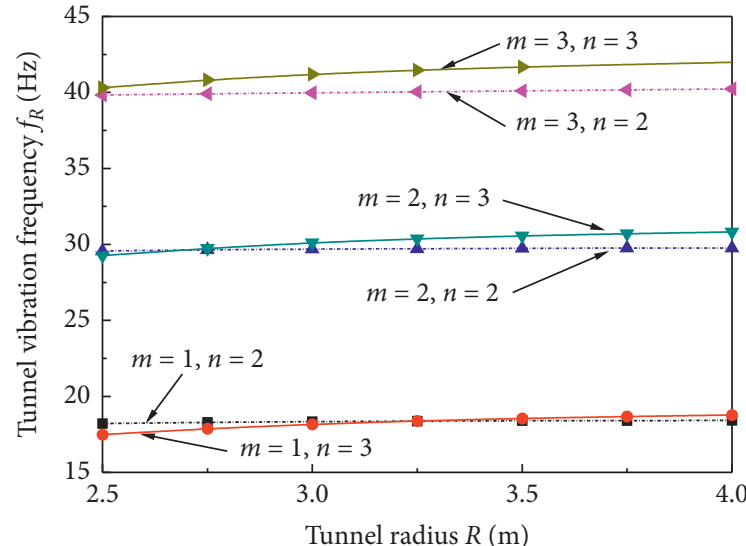

FIGURE 4: Relationship between natural frequency of the tunnel and changes in the tunnel radius change for different modes, where $\eta=1.0 \times 10^{6} \mathrm{~Pa} \cdot \mathrm{s}$.

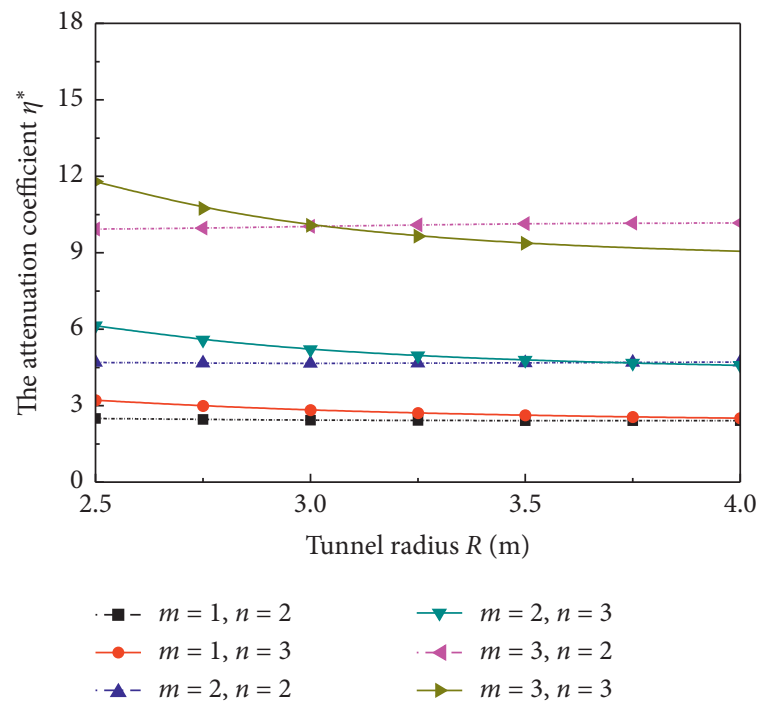

Figure 5: Relationship between attenuation and changes in the tunnel radius for different modes, where $\eta=1.0 \times 10^{6} \mathrm{~Pa} \cdot \mathrm{s}$.

\subsection{Effect of Tunnel Wall Thickness on Frequency of Tunnel} Structure. Figure 6 shows the variations in the natural frequency of the tunnel structure at different wall thicknesses. Except for the tunnel wall thickness, the calculation parameters are the same as those given in Section 5.1, and $\eta=1.0 \times 10^{6} \mathrm{~Pa} \cdot \mathrm{s}$. As shown in Figure 6, with an increase in wall thickness, the natural frequency of the tunnel for each mode decreases. All modes with the same axial mode number $m$ have basically the same natural frequency and exhibit the same variation trend. When the tunnel wall thickness is at least $0.3 \mathrm{~m}$, the natural frequency of the tunnel for higher circumferential modes is lower at the same axial mode number. Figure 7 illustrates the variations in attenuation due to the tunnel structure at different wall thicknesses for each mode. The attenuation coefficient increases with an increase in tunnel wall thickness. When the tunnel wall thickness is at least $0.25 \mathrm{~m}$, the attenuation of each mode increases with an increase in tunnel wall thickness, and at the same axial mode number the attenuation coefficient for a

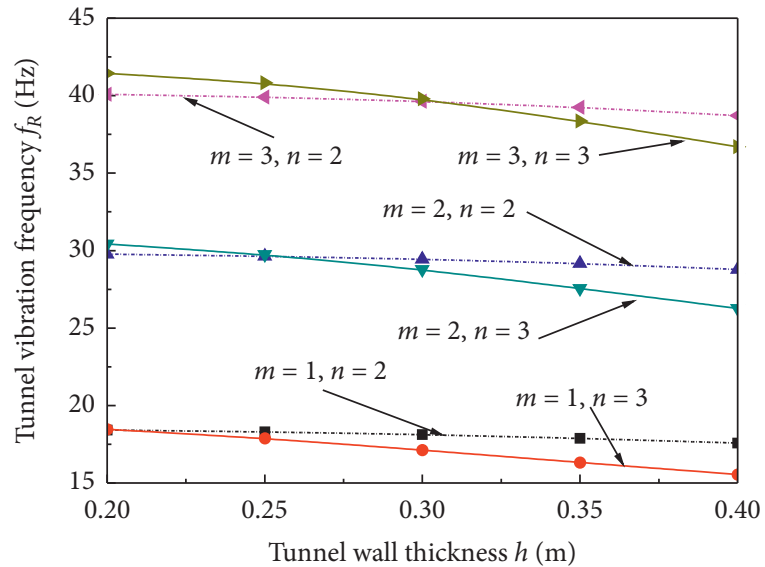

FIGURE 6: Relationship between natural frequency of the tunnel and tunnel wall thickness for different modes, where $\eta=1.0 \times 10^{6} \mathrm{~Pa} \cdot \mathrm{s}$.

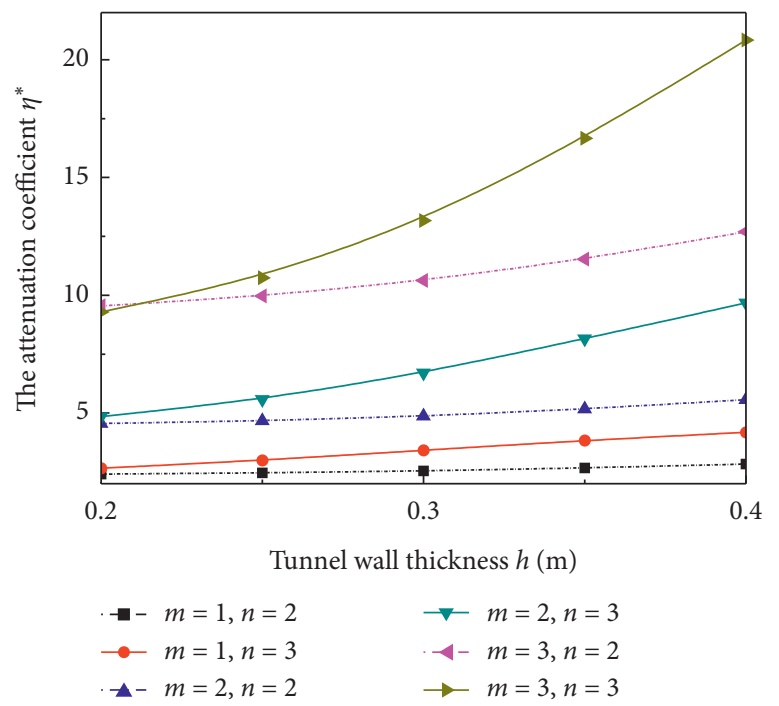

Figure 7: Relationship between attenuation and tunnel wall thickness for different modes, where $\eta=1.0 \times 10^{6} \mathrm{~Pa} \cdot \mathrm{s}$.

high circumferential mode number is higher. This indicates that in a viscous soil medium the influence of an increase in tunnel wall thickness on the natural vibration frequency of the tunnel structure is mainly caused by an increase in the generalized mass of the tunnel.

5.5. Effect of Tunnel Length on Frequency of Tunnel Structure. Figures 8 and 9 show the variations in the natural frequency of the tunnel and attenuation due to the tunnel structure at different tunnel length. Except for the length of the tunnel, the calculation parameters are the same as those given in Section 5.1, and $\eta=1.0 \times 10^{6} \mathrm{~Pa}$.s. Under the influence of coupling with viscous soil, the natural frequency of the tunnel and attenuation due to the tunnel significantly decrease with an increase in tunnel length, and at the same axial mode number the natural frequency of the tunnel for a high circumferential mode number is lower. As shown in Figure 9, with an increase in tunnel length the attenuation due to the tunnel tends to be constant. This 


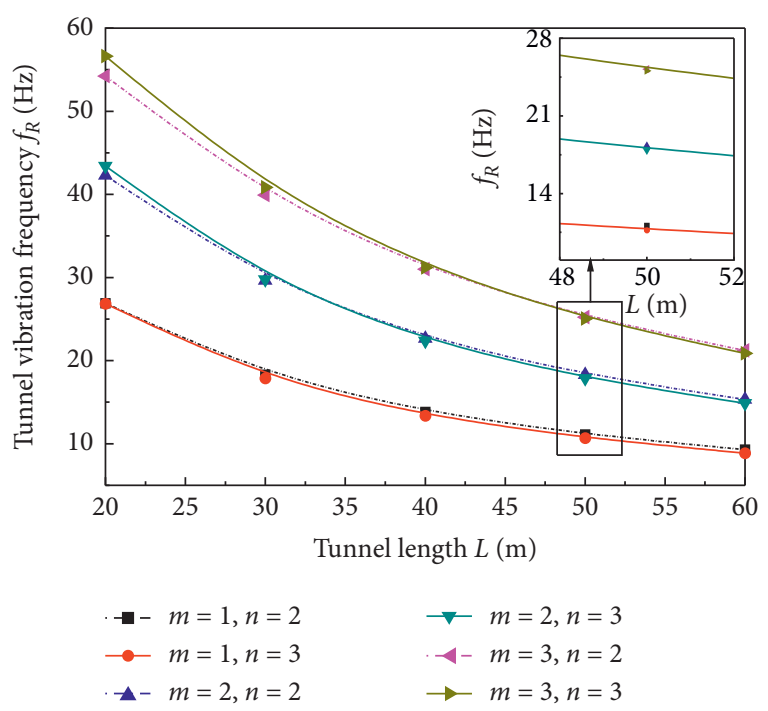

FIGURE 8: Relationship between natural frequency of the tunnel and tunnel length for different modes, where $\eta=1.0 \times 10^{6} \mathrm{~Pa} \cdot \mathrm{s}$.

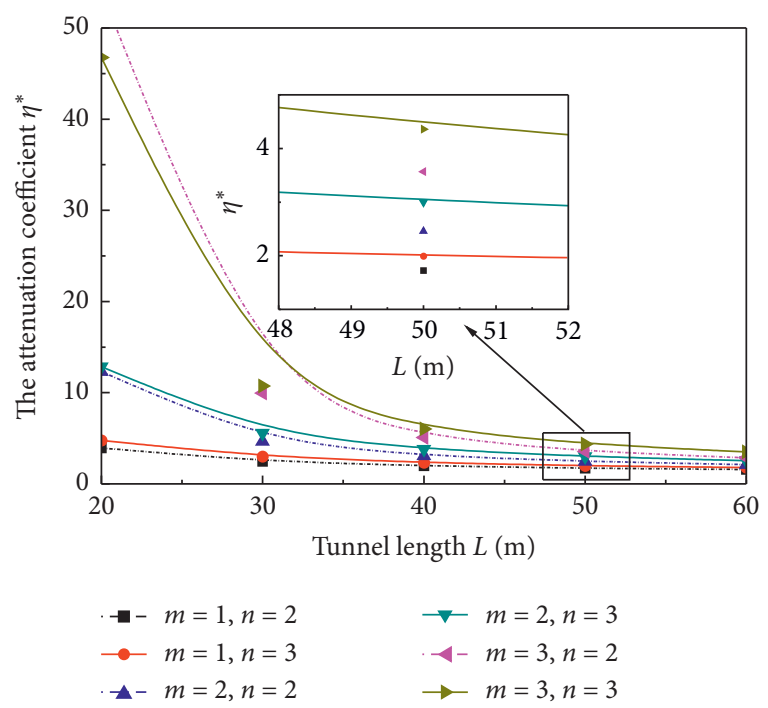

FIGURE 9: Relationship between attenuation and tunnel length for different modes, where $\eta=1.0 \times 10^{6} \mathrm{~Pa} \cdot \mathrm{s}$.

indicates that in a viscous soil medium the effect of an increase in tunnel length on the natural vibration frequency of the tunnel structure is mainly caused by decrease in the generalized stiffness of the structure.

\subsection{Effect of Tunnel Depth on Frequency of Tunnel Structure.} In this paper, the change of soil modulus is used to measure the buried depth of the tunnel, and the influence of buried depth on vibration frequency of the tunnel is studied. Except for the modulus of soil, the calculation parameters are the same as those given in Section 5.1, and $\eta=1.0 \times 10^{6} \mathrm{~Pa} \cdot \mathrm{s}$. As shown in Figure 10, with an increase in the modulus of soil, the natural frequency of the tunnel for each mode increases. All modes with the same axial mode number $m$ have basically the same natural frequency and exhibit the same variation trend. Figure 11 shows the attenuation curves for each mode

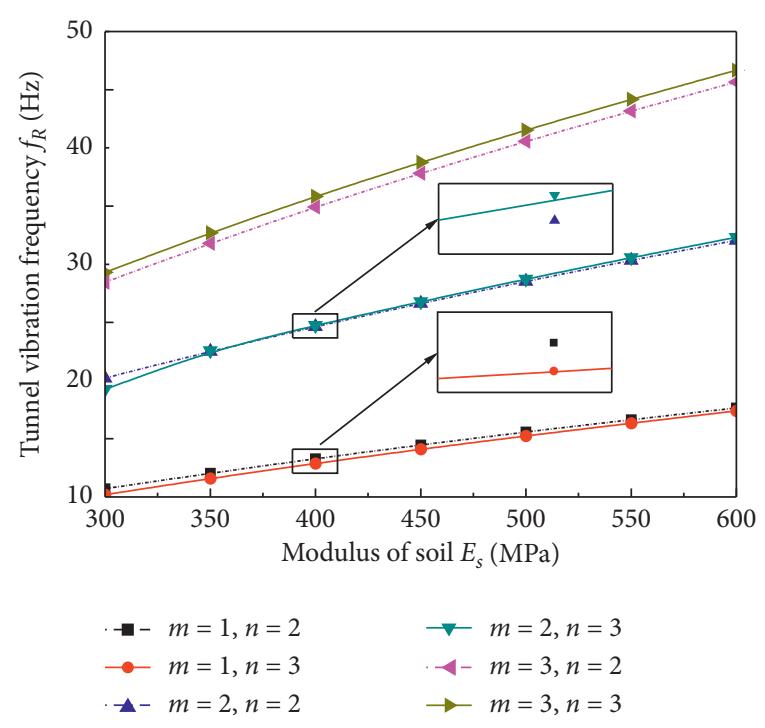

FIGURE 10: Relationship between natural frequency of the tunnel and the modulus of soil, where $\eta=1.0 \times 10^{6} \mathrm{~Pa} \cdot \mathrm{s}$.

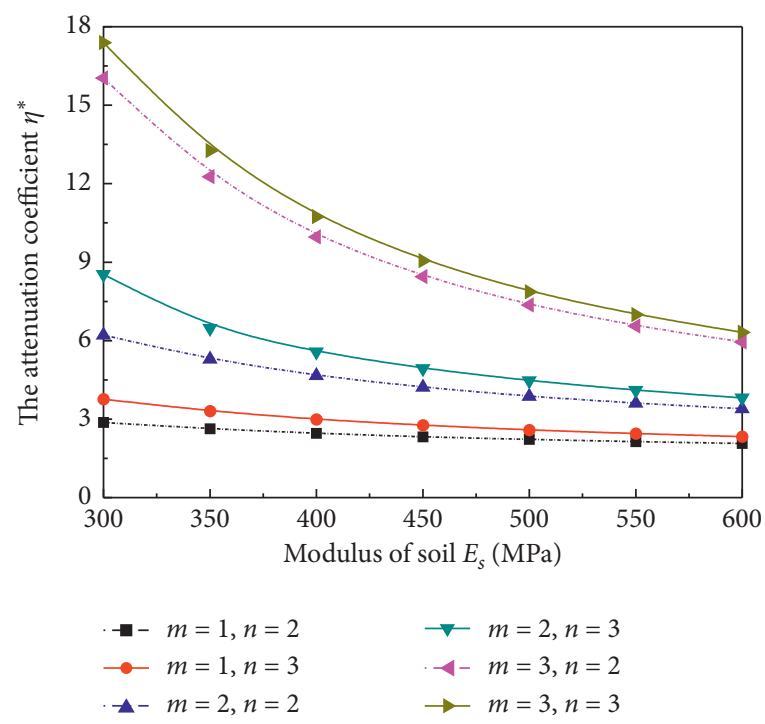

FIGURE 11: Relationship between attenuation and the modulus of soil, where $\eta=1.0 \times 10^{6} \mathrm{~Pa} \cdot \mathrm{s}$.

corresponding to Figure 10. It can be considered that, with the increase of the buried depth of the tunnel, the influence of soil media on the vibration characteristics of the tunnel structure is mainly the influence of additional stiffness.

\section{Conclusions}

In this paper, the vibration control equation for the coupling of a tunnel and viscous soil is established by using the theory of moderately thick cylindrical shells and the method of wave propagation and representing the soil around the tunnel in simplified form as an isotropic viscoelastic medium. The effect of a viscous soil medium on the natural vibration frequency of the tunnel structure is mainly manifested as the effect of additional mass. For each mode, 
the natural frequency of the tunnel structure decreases with an increase in the viscosity of the soil around the tunnel, and attenuation increases with an increase in soil viscosity. Higher modes are more sensitive to soil viscosity than are lower modes.

In a viscous soil medium, the natural frequency of the tunnel structure decreases with the increase of tunnel wall thickness, tunnel length, and tunnel buried depth. When the tunnel radius is large, the natural frequency of the tunnel structure slightly increases with an increase in the tunnel radius.

\section{Data Availability}

All data, models, and code generated or used during the study appear in the submitted article.

\section{Conflicts of Interest}

The authors declare that they have no conflicts of interest.

\section{Acknowledgments}

The research described in this paper was financially supported by the National Natural Science Foundation of China $(11972285,11872300$, and 11572245) and the Natural Science Foundation of Shaanxi Province (no. 2013JQ1008). This support is gratefully acknowledged.

\section{References}

[1] X. M. Zhang, G. R. Liu, and K. Y. Lam, "Coupled vibration analysis of fluid-filled cylindrical shells using the wave propagation approach," Applied Acoustics, vol. 62, no. 3, pp. 229-243, 2001.

[2] J. A. Forrest and H. E. M. Hunt, "A three-dimensional tunnel model for calculation of train-induced ground vibration," Journal of Sound and Vibration, vol. 294, no. 4-5, pp. 678-705, 2006.

[3] J. A. Forrest and H. E. M. Hunt, "Ground vibration generated by trains in underground tunnels," Journal of Sound and Vibration, vol. 294, no. 4-5, pp. 706-736, 2006.

[4] S. Sun, D. Cao, and S. Chu, "Free vibration analysis of thin rotating cylindrical shells using wave propagation approach," Archive of Applied Mechanics, vol. 83, no. 4, pp. 521-531, 2013.

[5] I. D. Moore and F. Guan, "Three-dimensional dynamic response of lined tunnels due to incident seismic waves," Earthquake Engineering \& Structural Dynamics, vol. 25, no. 4, pp. 357-369, 1996.

[6] D. Clouteau, M. Arnst, T. M. Al-Hussaini, and G. Degrande, "Freefield vibrations due to dynamic loading on a tunnel embedded in a stratified medium," Journal of Sound and Vibration, vol. 283, no. 1-2, pp. 173-199, 2005.

[7] G. Degrande, D. Clouteau, R. Othman et al., "A numerical model for ground-borne vibrations from underground railway traffic based on a periodic finite element-boundary element formulation," Journal of Sound and Vibration, vol. 293, no. 3-5, pp. 645-666, 2006.

[8] S. Gupta, M. F. M. Hussein, G. Degrande, H. E. M. Hunt, and D. Clouteau, "A comparison of two numerical models for the prediction of vibrations from underground railway traffic,"
Soil Dynamics and Earthquake Engineering, vol. 27, no. 7, pp. 608-624, 2007.

[9] M. F. M. Hussein, S. Gupta, H. E. M. Hunt et al., "An efficient model for calculating vibration from a railway tunnel buried in a half-space," in Proceedings of the 13th International Congress on Sound and Vibration, Vienna, Austria, June 2006.

[10] K. A. Kuo, H. E. M. Hunt, and M. F. M. Hussein, "The effect of a twin tunnel on the propagation of ground-borne vibration from an underground railway," Journal of Sound and Vibration, vol. 330, no. 25, pp. 6203-6222, 2011.

[11] A. Clot, R. Arcos, J. Romeu, and T. Pàmies, "Dynamic response of a double-deck circular tunnel embedded in a fullspace," Tunnelling and Underground Space Technology, vol. 59, pp. 146-156, 2016.

[12] G. Parry, S. Summers, and D. Yates, "Validation of the pipe in pipe vibration software model to determine ground-borne noise and vibration levels above construction tunnels and the determination of end corrections for different train operating scenarios," The Journal of the Acoustical Society of America, vol. 135, no. 4, p. 2288, 2014.

[13] W. Yang, M. F. M. Hussein, and A. M. Marshall, "Centrifuge and numerical modelling of ground-borne vibration from an underground tunnel," Soil Dynamics and Earthquake Engineering, vol. 51, pp. 23-34, 2013.

[14] T. Senjuntichai, S. Mani, and R. K. N. D. Rajapakse, "Vertical vibration of an embedded rigid foundation in a poroelastic soil," Soil Dynamics \& Earthquake Engineering, vol. 26, no. 67, pp. 626-636, 2006.

[15] R. Kumar, A. Miglani, and L. Debnath, "Radial displacements of an infinite liquid saturated porous medium with cylindrical cavity," Computers \& Mathematics with Applications, vol. 37, no. 7, pp. 117-123, 1999.

[16] S. M. Hasheminejad and H. Hosseini, "Dynamic stress concentration near a fluid-filled permeable borehole induced by general modal vibrations of an internal cylindrical radiator," Soil Dynamics and Earthquake Engineering, vol. 22, no. 6, pp. 441-458, 2002.

[17] S. M. Hasheminejad and M. Komeili, "Effect of imperfect bonding on axisymmetric elastodynamic response of a lined circular tunnel in poroelastic soil due to a moving ring load," International Journal of Solids and Structures, vol. 46, no. 2, pp. 398-411, 2009.

[18] G. Y. Gao, J. F. He, and L. Jia, "Dynamic response induced by running subway in saturated ground," Journal of Zhejiang University (Engineering Science Edition), vol. 44, no. 10, pp. 1925-1930, 2010.

[19] C. He, S. H. Zhou, H. G. Di, and Y. Shan, “A 2.5-D coupled FE-BE model for the dynamic interaction between saturated soil and longitudinally invariant structures," Computers and Geotechnics, vol. 82, pp. 211-222, 2017.

[20] S. Zhou, C. He, and H. Di, "Dynamic 2.5-D green's function for a poroelastic half-space," Engineering Analysis with Boundary Elements, vol. 67, pp. 96-107, 2016.

[21] H. Di, S. Zhou, C. He, X. Zhang, and Z. Luo, “Three-dimensional multilayer cylindrical tunnel model for calculating train-induced dynamic stress in saturated soils," Computers and Geotechnics, vol. 80, pp. 333-345, 2016.

[22] J. F. Lu and D. S. Jeng, "Dynamic analysis of an infinite cylindrical hole in a saturated poroelastic medium," Archive of Applied Mechanics, vol. 76, no. 5-6, pp. 263-276, 2006.

[23] J.-F. Lu and D.-S. Jeng, "A half-space saturated poro-elastic medium subjected to a moving point load," International Journal of Solids and Structures, vol. 44, no. 2, pp. 573-586, 2007. 
[24] H. X. Gao and M. J. Wen, "Frequency responses of a partial sealed tunnel with fractional derivative viscoelastic lining," Acta Mechanica Solida Sinica, vol. 33, no. 6, pp. 637-643, 2012.

[25] Z. Yuan, Y. Cai, and Z. Cao, "An analytical model for vibration prediction of a tunnel embedded in a saturated fullspace to a harmonic point load," Soil Dynamics and Earthquake Engineering, vol. 86, pp. 25-40, 2016.

[26] Z. Yuan, Z. Cao, A. Boström, and Y. Cai, "The influence of pore-fluid in the soil on ground vibrations from a tunnel embedded in a layered half-space," Journal of Sound and Vibration, vol. 419, pp. 227-248, 2018.

[27] S. Jones and H. Hunt, "Voids at the tunnel-soil interface for calculation of ground vibration from underground railways," Journal of Sound and Vibration, vol. 330, no. 2, pp. 245-270, 2011.

[28] N. Yasuda, K. Tsukada, and T. Asakura, "Elastic solutions for circular tunnel with void behind lining," Tunnelling and Underground Space Technology, vol. 70, pp. 274-285, 2017.

[29] Y. Huang, H. R. Huang, F. S. He et al., The Linear Theory of Elastic Shells, Science Press, Beijing, China, 2007.

[30] K. F. Graff, Wave Motion in Elastic Solids, Oxford University Press, Oxford, UK, 1975.

[31] D. C. Gazis, "Three-dimensional investigation of the propagation of waves in hollow circular cylinders. I. analytical foundation," The Journal of the Acoustical Society of America, vol. 31, no. 5, pp. 568-573, 1959. 